\title{
The Multisystem Inflammatory Syndrome of Childhood (MIS-C)
}

\author{
Reza Sinaei ${ }^{1}$ - Ali Hosseininasab ${ }^{2} \cdot$ Maedeh Jafari $^{3} \cdot$ Shahryar Eslami $^{4} \cdot$ Saeedeh Parvaresh ${ }^{5}$
}

Received: 13 September 2020 / Accepted: 10 December 2020/Published online: 12 February 2021

(C) Dr. K C Chaudhuri Foundation 2020

To the Editor: Although COVID-19 seems to be milder in children than adults, the SARS-CoV-2 now is showing the ugliest faces off from itself [1,2]. Recently, a severe and novel entity has emerged in children as a multi-system inflammatory syndrome. Here we report a series of affected children in southeast of Iran [3-5].

From June 1 to August 12 of 2020, 15 pediatric patients with Kawasaki like features and a wide spectrum of clinical findings were admitted at Kerman city Afzalipour Hospital in the southeast of Iran. Four of them were more violent cases of SARS$\mathrm{CoV}-2$ infection at the end of the spectrum as MIS-C demonstrations, with positive PCR and/or anti-SARS-CoV-2 antibody results. These patients were admitted at PICU and their main symptoms were tachycardia and hypotension. One of them had an increasing pro-BNP $\geq 35,000 \mathrm{pg} / \mathrm{mL}$ but troponin and CPK$\mathrm{MB}$ were in normal ranges. All patients with MIS-C had severe cardiac dysfunction and reduced ejection fraction (mean 20\%). They received intravenous PDE3 inhibitor (milrinone) and intravenous epinephrine, for an average of $5 \mathrm{~d}$. The electrocardiogram had nonspecific changes such as sinus tachycardia and abnormal ST-T changes. All patients received IVIG $2 \mathrm{~g} / \mathrm{kg}$, and all in adjacent to MIS-C and four in Kawasaki like spectrum received glucocorticoids. The mean age of our patients was 49.6

\section{Maedeh Jafari}

mjafari@kmu.ac.ir

1 Department of Pediatrics, School of Medicine, Endocrinology and Metabolism Research Center, Institute of Basic and Clinical Physiology Sciences, Kerman University of Medical Sciences, Kerman, Iran

2 Department of Pediatric Infectious Disease, Infectious and Tropical Research Center, Kerman University of Medical Sciences, Kerman, Iran

3 Department of Pediatrics, School of Medicine, Clinical Research Development Unit, Afzalipour Hospital, Kerman University of Medical Sciences, Kerman, Iran

4 Department of Pediatrics Infectious Disease, Kerman University of Medical Sciences, Kerman, Iran

5 Department of Pediatrics, School of Medicine, Kerman University of Medical Sciences, Kerman, Iran mo and they had a wide array of manifestations of persistent fever, rash, diarrhea, vomiting, moderate to severe heart failure, shock similar to toxic shock syndrome, and relative delay arthritis. Four patients with MIS-C had either moderate to severe heart failure or presented with shock, requiring the vasopressor and resuscitating therapies. The electrocardiogram had nonspecific changes such as sinus tachycardia and abnormal ST-T changes. All patients received IVIG $2 \mathrm{~g} / \mathrm{kg}$, and all in adjacent to MIS-C and four in Kawasaki-like spectrum received glucocorticoids. The average length of PICU stay was $6 \mathrm{~d}$, and all patients were discharged in good condition after improving their clinical symptoms. Although most patients with this syndrome are ill and have heart problems and need PICU stay, they can recover using appropriate treatment.

\section{Compliance with Ethical Standards}

Ethics Approval This study had been approved by the ethics committee of Kerman University of Medical Sciences (Code: IR.KMU.AH.REC.1399.064).

Conflict of Interest None.

\section{References}

1. Lu X, Zhang L, Du H, et al. SARS-CoV-2 infection in children. New Engl J Med. 2020;382:1663-5.

2. Riphagen S, Gomez X, Gonzalez-Martinez C, Wilkinson N, Theocharis P. Hyperinflammatory shock in children during COVID-19 pandemic. Lancet. 2020;395(10237):1607-8.

3. Verdoni L, Mazza A, Gervasoni A, et al. An outbreak of severe Kawasaki-like disease at the Italian epicentre of the SARS-CoV-2 epidemic: an observational cohort study. Lancet. 2020;395:1771-8.

4. Hosseninasab A, Shafiei Bafti M, Ebrahimi S, et al. Coronavirus disease 2019 in children with acute respiratory infection: a study from southeastern Iran, Shiraz E-Med J. Online ahead of Print; In Press (In Press):e108377. https://doi.org/10.5812/semj.108377.

5. Chiotos K, Bassiri H, Behrens EM, et al. Multisystem inflammatory syndrome in children during the coronavirus 2019 pandemic: a case series. J Pediatric Infect Dis Soc. 2020;9(3):393-8.

Publisher's Note Springer Nature remains neutral with regard to jurisdictional claims in published maps and institutional affiliations. 